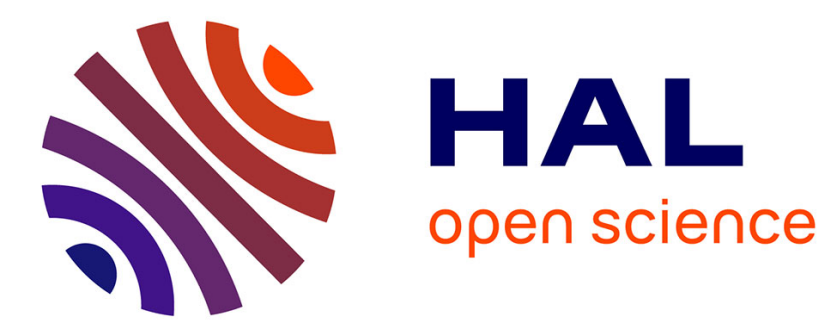

\title{
THE WAVELENGTH-RATIO METHOD : A NOVEL APPROACH TO EXTINCTION-FREE DETERMINATION OF FM/FN
}

\author{
J. Guigay, M. Schlenker, J. Baruchel
}

\section{- To cite this version:}

J. Guigay, M. Schlenker, J. Baruchel. THE WAVELENGTH-RATIO METHOD: A NOVEL APPROACH TO EXTINCTION-FREE DETERMINATION OF FM/FN. Journal de Physique Colloques, 1982, 43 (C7), pp.C7-107-C7-111. 10.1051/jphyscol:1982716 . jpa-00222323

HAL Id: jpa-00222323

https://hal.science/jpa-00222323

Submitted on 1 Jan 1982

HAL is a multi-disciplinary open access archive for the deposit and dissemination of scientific research documents, whether they are published or not. The documents may come from teaching and research institutions in France or abroad, or from public or private research centers.
L'archive ouverte pluridisciplinaire HAL, est destinée au dépôt et à la diffusion de documents scientifiques de niveau recherche, publiés ou non, émanant des établissements d'enseignement et de recherche français ou étrangers, des laboratoires publics ou privés. 


\title{
THE WAVELENGTH-RATIO METHOD : A NOVEL APPROACH TO EXTINCTION-FREE DETERMINATION OF $F_{M} / F_{N}$
}

\author{
J.P. Guigay, M. Schlenker ${ }^{*}$ and J. Baruche ${ }^{*}$ \\ DRE/CENG and USMG, $85 \mathrm{X}, 38041$ Grenobze Cedex, Erance \\ *Laboratoire Louis Néel, CNRS-USMG, $166 \mathrm{X}, 38042$ Grenobze Cedex, France
}

\begin{abstract}
Résumé . - Les variations, en fonction de la longueur d'onde, des réflectivités $\rho_{+}$ et $\rho_{-}$correspondant aux deux polarisations des neutrons incidents devraient, pour un monocristal magnétique en forme de plaque, être liées par une loi d'échelle indépendante de l'extinction. Cette propriẻté permettrait, moyennant vérification expérimentale, d'obtenir très directement le rapport des composantes magnétiques et nucléaires des facteurs de structure, sans avoir à efectuer des corrections d'extinction.

Abstract.- The variations with wavelength of the reflectivities $\rho_{+}$and $\rho_{-}$corresponding to the two polarization states of the incident neutrons should be related through an extinction - independent scaling law in the case of a plate-shaped crystal. If borne out by experiment, this property should provide a very direct way of measuring the ratio of the magnetic and nuclear contributions to the structure factor, without use of extinction corrections.
\end{abstract}

\section{Introduction}

The structure factor $F(\vec{h})$ of a magnetic crystal with colinear magnetic structure depends on the relative orientations of the scattering vector $\vec{h}$, the crystal magnetization $\vec{M}$ and the neutron beam polarization $\vec{P}$. In the simplest case,

$$
\mathrm{F}_{ \pm}(\overrightarrow{\mathrm{h}})=\mathrm{F}_{\mathrm{N}}(\overrightarrow{\mathrm{h}}) \pm \mathrm{F}_{\mathrm{M}}(\overrightarrow{\mathrm{h}})
$$

when $\vec{M}$ is perpendicular to $\overrightarrow{\mathrm{h}}$ and $\overrightarrow{\mathrm{P}}$ is either parallel or antiparallel to $\overrightarrow{\mathrm{M}}, \mathrm{F}_{\mathrm{N}}$ and $\mathrm{F}_{\mathrm{M}}$ being the nuclear and magnetic structure factors respectively.

In the following, our considerations are limited to a fixed diffraction vector. The accurate determination of $F_{M}$ is of great interest for determining the magnetization distribution in the unit cell of the crystal ; it implies the measurement of the ratio $\left(F_{+} / F_{-}\right)$.

An experimentally convenient quantity is the ratio $R=\left(I_{+} / I_{-}\right)$of the diffracted intensities $I_{+}$and $I_{-}$corresponding to the ( \pm ) polarization states. In the very favourable case of an ideally imperfect crystal (kinematical scattering), this so-called "flipping ratio" $R$ is independent of the crystal thickness $t$ and of the neutron wavelength $\lambda$, and is simply equal to $R_{K}=\left(F_{+} / F_{-}\right)^{2}$.

However, extinction corrections (non ideally imperfect crystal case) are often necessary; for instance, the presence of primary extinction can result in a wavelength dependence of $\mathrm{R}$; a particular test for the presence of secondary extinction is a variation of $R$ as the crystal is rotated around its Bragg peak position [1]. Then :

$$
\mathrm{R}=\mathrm{R}_{\mathrm{K}} \frac{\mathrm{y}_{+}}{\mathrm{y}_{-}}
$$

$y_{+}$and $y_{-}$being the extinction coefficients applied to the ( \pm ) diffracted intensities. The determination of the ratio $\left(y_{+} / y_{-}\right)$. is often not an easy task. The crystal is assumed to have a mosaïc structure described by some model with phenomenological parameters (statistical distribution of crystallite size and misorientation), the values of which are fitted to experimental data allowing the calculation of the 
extinction coefficients. A very complete study of extinction effects has been performed by Bonnet, Delapalme, Becker and Fuess [2] in the case of ferrimagnetic Yttrium-Iron Garnet (YIG). Extinction corrections are large (and therefore delicate) in this case because the available crystals are far from the ideally imperfect structure. It is even possible to observe unusual wave-dynamical effects : we have measured the flipping ratio $R$ for different values of the neutron wavelength $\lambda$ from a carefully chosen YIG-crystal, in symmetrical Lave geometry and obtained an osci1latory behaviour of $R(\lambda)$; this experiment is reported in another paper of this conference $[3]$.

The method we shall propose for the determination of $\left(F_{+} / F_{-}\right)$is much more straightforward in principle, because it is basically independent of the amount of extinction. This will be made clear by considering the limiting case of an ideally perfect crystal described by the wave-dynamical theory.

2. Integrated reflectivities for perfect and ideally imperfect crystals.

We consider a perfect non-absorbing crystal, having the form of a plane parallel plate, magnetically saturated in a direction parallel to the reflecting planes, in which case the usual dynamical theory for scalar waves is applicable to the $(+)$ and $(-)$ polarized beams separately ; the $(+)$ and $(-)$ indexes will be omitted when they are not necessary. is $|3|$ :

$$
\rho=\frac{\lambda}{\sin \left(2 \theta_{B}\right)} \times \frac{\lambda|F|}{V_{c}} \times W\left(\frac{\lambda t|F|}{V_{C} \sqrt{\gamma_{O} \gamma_{h}}}\right)
$$

$\mathrm{V}_{\mathrm{C}}$ being the volume of the unit cell, $\theta_{\mathrm{B}}$ the Bragg angle, $t$ the crystal thickness $\psi_{O}^{c}$ and $\gamma_{h}$ the components of unit vectors along the incident and reflected directions on the direction normal to the crystal surfaces (in the symmetrical laue geometry $\left.\gamma_{0}=\gamma_{h}=\cos \theta_{B}\right)$. W(x) is the Waller function (fig.l):

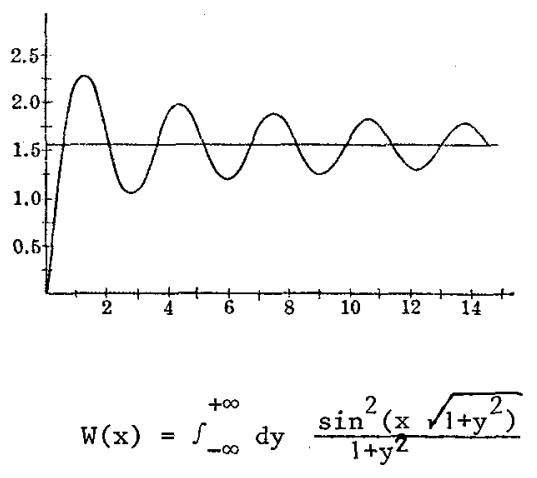

Fig. 1 - Plot of the Waller function W(x)

which can be expressed in terms of Bessel functions :

$$
W(x)=\frac{\pi}{2} \int_{0}^{2 x} d u \quad J_{0}(u)=\pi \quad \sum_{n=0}^{\infty} J_{2 n+1}^{J}(2 x)
$$

In the case of Bragg geometry (reflection case) formula (2) is replaced by :

$$
\rho=\pi \frac{\lambda}{\sin \left(2 \theta_{B}\right)} \frac{\lambda|F|}{V_{c}} \times \operatorname{Tanh}\left(\frac{\lambda t|F|}{V_{c} \sqrt{\gamma_{o} \gamma_{h}}}\right)
$$

for the symmetrical Bragg geometry $\gamma_{0}=\gamma_{h}=\sin \theta_{B}$ 
It is convenient, following Zachariasen [4], to define:

$$
A=\frac{\lambda t|F|}{V_{c} \sqrt{Y_{0} Y_{h}}}=\pi \frac{t}{\Lambda}
$$

and to express $p$ as :

$$
\rho=\frac{d}{t} \frac{\sqrt{\gamma_{0} \gamma_{h}}}{\cos _{B}} \quad A \quad \times\left\{\begin{array}{l}
W(A) \quad \text { (Laue geometry) } \\
\pi \cdot \tanh (A) \text { (Bragg geometry) }
\end{array}\right.
$$

$\mathrm{d}=\lambda /\left(2 \sin \theta_{\mathrm{B}}\right)$ being the reflecting planes spacing and $\Lambda$ the extinction distance.

The kinematical expression, applicable to ideally imperfect crystals, is obtained as the limit of ( 7 ) for $A \rightarrow 0$

$$
\rho_{\mathrm{K}}=\pi \frac{\mathrm{d}}{\mathrm{t}} \times \frac{\sqrt{\gamma_{\mathrm{O}} \gamma_{\mathbf{h}}}}{\cos \theta_{B}} \times \mathrm{A}^{2}
$$

for both Laue and Bragg geometries.

\section{Fundamental scaling relation}

The preceding formulas are applicable to the $\rho_{+}$and $\rho_{-}$reflectivities from a magnetic crystal for the $(+)$ and $(-)$ beam polarization states.

We point out that the variation of $A$, when the beam polarization is reversed $\left(F_{+} \rightarrow F_{-}\right.$or inversely), can be formally compensated by a supplementary change of the crystal thickness $t$ or of the neutron wavelength $\lambda$; a change of $\lambda$ seems the most satisfactory choice.

In the case of Laue symmetrical geometry, $\rho_{+}$and $\rho_{-}$which depend on $\lambda$ through

$$
u=\lambda / \cos \theta_{B}=2 d \operatorname{tg} \theta_{B}
$$

,will be considered as functions $\rho_{ \pm}(u)$; they satisfy the scaling relation :

$$
\rho_{+}(u)=\rho_{-}\left(u\left|\frac{F_{+}}{F_{-}}\right|\right)
$$

which is fundamental to our purpose, as the scaling factor is just the quantity we are interested in.

This scaling relation holds in the Laue or Bragg non-symmetrical cases, by considering :

$$
\mathbf{u}=\lambda / \sqrt{\gamma_{\mathrm{o}} \gamma_{\mathrm{h}}}
$$

instead of (9) ; however, according to (7), the integrated reflectivities also depend on $\lambda$ through $\left(\sqrt{\gamma_{0} \gamma_{h}} / \cos \theta\right)$; it is thus necessary to re-define the $\rho_{ \pm}$functions of (10) : for each wavelength $\lambda$, the integrated reflectivities must be multiplied by $\left(\cos \theta_{B} /{\sqrt{\gamma_{O} \gamma_{h}}}\right)$

In the particular case of symetrical Bragg geometry, formula (6) shows that A does not depend on $\lambda$, because $\sqrt{\gamma_{0} \gamma_{h}}$ is then equal to $\sin \theta_{B}$. This shows that our method described below is not applicable to this case and to non-symetrical Bragg geometry with a low degree of asymmetry.

We know that the scaling relation (10) holds in the two limiting cases (perfect and ideally imperfect crystals) ; we shall assume that it also holds in intermediate cases, that is for real crystals. This is equivalent to assuming that :

$$
\rho=\frac{\sqrt{\gamma_{0} \gamma_{h}}}{\cos \theta_{B}} \frac{d}{t} F_{(A)}
$$

where $\mathcal{F}(A)$ is a one-variable function characteristic of the considered crystal, the knowledge of the form of this function being unnecessary for our purpose. 


\section{Discussion of the proposed method.}

Measurements of the $( \pm)$ reflectivities for a continuous range of neutron-wavelengths will provide an experimental test of the scaling relation (10) and, if this relation appears to be actually verified, the value of the scaling factor equal to $\left|F_{+} / F_{-}\right|$. This method would thus be self-consistent.

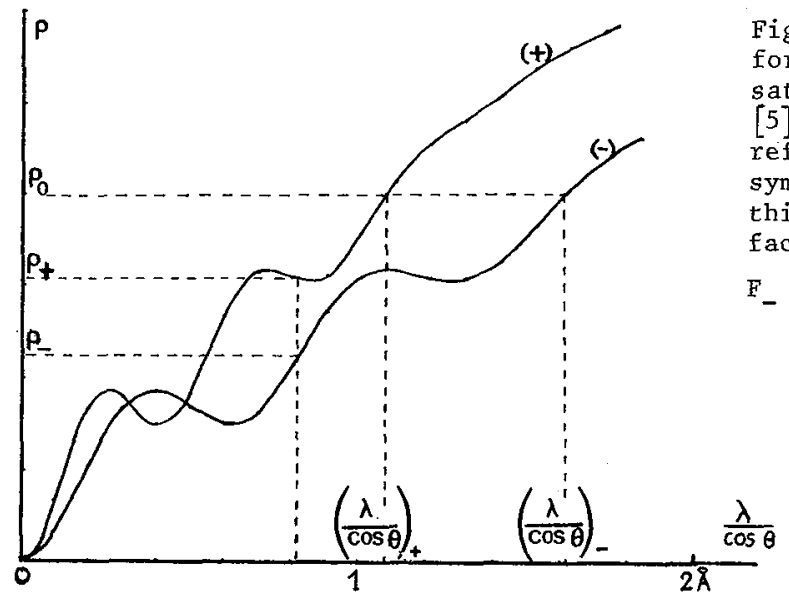

Fig.2 - Calculated reflectivities for the two neutron polarization sates, based on Kato's formulation $[5](E=0.9 ; \tau=50 \mu \mathrm{m}) ; 2 \overline{2} 0$ reflection from a YIG crystal in symmetrical Laue geometry; crystal thickness $0.48 \mathrm{~mm}$ ) ; structure factors from $[2]\left(\mathrm{F}_{+}=25.86\right.$; $F_{-}=16.74 \times 10^{-12} \mathrm{~cm}$ )

According to the graphical representation of fig. 2 , the usual flipping ratio, which is the ratio of the $\rho$ values defined by a vertical line, is replaced by the "wavelength-ratio", which is the ratio of the $\left(\lambda / \sqrt{\gamma_{o} \gamma_{h}}\right)$ values defined by horizontal lines and directly provides $\left|F_{+} / F_{-}\right|$.

The basic hypothesis formulated by (10) or (11) seems to be in agreement with the statistical diffraction theory developed by Rato [5.]; Kato's theory contains 2 new parameters, a "static Debye-Waller Factor" $E$ and a coherence length $\tau$. The scaling relation (10) holds if these parameters, which are characteristics of the crystal defects and of the reflection under consideration, do not depend on $\lambda$; this seems a reasonable assumption. Actually, the validity of our treatment should also be considered from the point of view of other extinction theories. This point is presently being investigated. However, we do not really need a theoretical justification of the fundamental scaling relation, as its validity or non-validity is supposed to be checked by the experiment itself.

We should like to point out some difficulties of our method. On the one hand comparing reflectivity measurements for different neutron wavelengths is more difficult than flipping ratio measurements, because the intensity and characteristics (angular and wavelength spreads) of the incoming beam are modified when the wavelength is changed. On the other hand, if $\left|F_{+} / F_{-}\right|$is very different from unity, the available range of neutron wavelengths may not be large enough to apply the scaling relation (10). Last, this technique is not applicable to the symmetrical Bragg geometry.

But this method should have the advantage, which makes it very valuable for some investigations, to be extinction-free. We intend to perform an experimental verification of the scaling relation (10) for wel1-characterized YIG crystals of different effective degrees of perfection; this will be interesting both from the point of view of extinction theory, and to measure accurately the magnetic structure factors. Applying the wavelength-ratio method to the preliminary results (fig.3) we obtained for the 220 reflection of the YIG-sample used in [3] yields $\left(F_{+} \neq F_{-}\right)=$ $1.60 \pm 0.10$, in fair agreement with previous measurements [2]. 


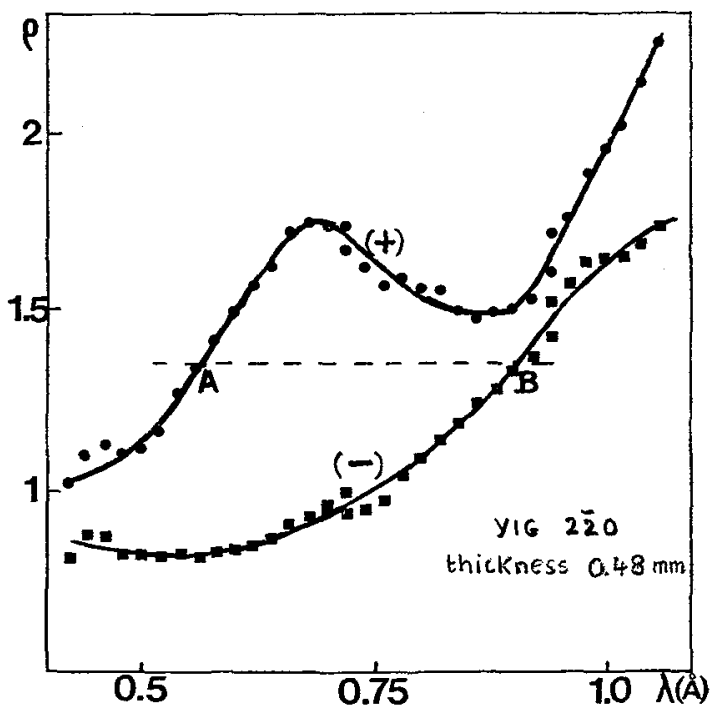

Fig.3 - Measured reflectivities (arbitrary units) as functions of neutron wavelength, corresponding to the calculated curves of Fig.2 ( $\lambda$ is practically equal to $\left.\lambda / \cos \theta_{B}\right)$. The scaling relation is well satisfied; the ratio of the slopes at the inflection. points $A$ and $B$ gives the ratio $F_{+} / F_{-} \simeq 1.6$

\section{References}

[1] Nathans R., Shuil C.G., Shirane G., Andresen A., J.Phys.Chem.Solids 10 (1959) 138.

[2] Bonnet M, Delapalme A., Becker P., Fuess H., Acta Cryst. A 32 (1976) 945 and J.Phys.Chem.Solids 40 (1979) 863.

[3] Baruche1 J., Guigay J.P., Mazuré-Espejo C., Schlenker M. and Schweizer J., paper presented at this conference.

[4] Zachariasen W.H., Theory of X-Ray diffraction in Crystals, Dover publication, (1967).

[5] Kato N., Acta Cryst. A36 (1980) 763 and 770. 